\title{
Sliding and Rocking of Unanchored Components and Structures: Chapter 7.6 ASCE 4 Revision 2
}

\section{Structures 2011 Congress}

U.S. Department of Energy

National Laboratory

operated by

Battelle Energy Alliance

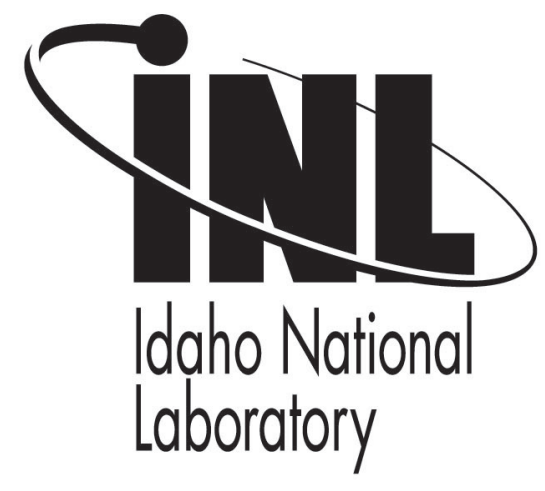

\section{S. R. Jensen \\ O. Gurbuz}

April 2011
This is a preprint of a paper intended for publication in a journal or proceedings. Since changes may be made before publication, this preprint should not be cited or reproduced without permission of the author. This document was prepared as an account of work sponsored by an agency of the United States Government. Neither the United States Government nor any agency thereof, or any of their employees, makes any warranty, expressed or implied, or assumes any legal liability or responsibility for any third party's use, or the results of such use, of any information, apparatus, product or process disclosed in this report, or represents that its use by such third party would not infringe privately owned rights. The views expressed in this paper are not necessarily those of the United States Government or the sponsoring agency. 


\title{
Sliding and Rocking of Unanchored Components and Structures: Chapter 7.6 ASCE 4 Revision 2
}

\author{
S. R. Jensen ${ }^{1}$ and O. Gurbuz ${ }^{2}$ \\ ${ }^{1}$ Idaho National Laboratory, Idaho Falls, ID, P. O. Box 1625, Idaho Falls, ID 83413- \\ 7101, Ph (208) 526-9137 stuart.jensen@inl.gov \\ ${ }^{2}$ Gurbuz Consulting, 16162 Tortola Circle, Huntington Beach, CA 92649, Ph (714) 625- \\ 0656,ogurbuz@aol.com
}

\begin{abstract}
Section 7.6 of ASCE 4-Rev 2, Seismic Analysis of Safety-Related Nuclear Structures: Standard and Commentary, provides updated guidance for analysis of rocking and sliding of unanchored structures and components subjected to seismic load. This guidance includes provisions both for simplified approximate energy-based approaches, and for detailed probabilistic time history analysis using nonlinear methods. Factors to be applied to the analytical results are also provided to achieve demand estimates at the $80 \%$ non-exceedance probability level. The present paper surveys the published literature supporting these provisions. The results of available testing and analyses are compared to results produced by both simplified and probabilistic approaches. In addition, adequacy of the standard's provisions for analysis methods and factors is assessed. A comparison is made between the achieved level of conservatism and the standard's nonexceedance probability target.
\end{abstract}

\section{BACKGROUND/INTRODUCTION}

Section 7.6 of ASCE 4-Rev 2, Seismic Analysis of Safety-Related Nuclear Structures: Standard and Commentary (ASCE, 2011), provides guidance for analysis of rocking and sliding of unanchored structures and components subjected to seismic load. Hereafter in this paper, "ASCE 4" shall refer to the draft standard, not to be confused with the existing ASCE 4-98. The guidance in ASCE 4 Section 7.6 is consistent with the current revision of ASCE/SEI 43-05, Seismi Design Criteria for Structures, Systems and Components in Nuclear Facilities (ASCE/SEI 43-05), but does provide some evolutionary updates as will be explained throughout this paper.

Seismic design and evaluation of nuclear structures can be divided into to two main tasks, which are each addressed by separate ASCE standards. Analysis of structures to determine seismic demands is governed by ASCE 4, while criteria to be used in the design of these structures are given by ASCE/SEI 43-05. The acceptability of the design is determined by the comparison of demands to capacities, as outlined in ASCE/SEI 43- 
05. These standards in combination are intended to satisfy a performance goal, described in ASCE/SEI 43-05 as to reasonably achieve both of the following criteria:

1. Less than about a $1 \%$ probability of unacceptable performance for the DBE ground motion

2. Less than about a $10 \%$ probability of unacceptable performance for a ground motion equal to $150 \%$ of the DBE ground motion.

(ASCE/SEI 43-05 Section 1.3)

Each standard provides a controlled degree of conservatism. ASCE 4-98 stated an intent to produce demands with a non exceedance probability (NEP) of approximately $90 \%$. The new draft of ASCE 4 will change that target to an NEP of approximately $80 \%$. ASCE/SEI 43-05 states an intent to produce capacities with an exceedance probability of approximately $98 \%$. These two margins, one on analysis and one on design, when combined are shown in the ASCE/SEI 43-05 commentary to satisfy the performance goal.

However, ASCE 4-98 did not contain provisions for rocking and sliding of unanchored components. Because the working group felt it would be useful to provide some guidance, ASCE/SEI 43-05 was issued including Section 7.1 containing provisions for evaluation of sliding and rocking of unanchored components. It was necessary under those circumstances to combine the conservatism for analysis and design into the design criteria standard. This conservatism is presented in ASCE/SEI 43-05 as factors of safety to be applied to the best-estimate (median centered) sliding distance or rocking angle to develop design values. These factors of safety were based upon judgment formed by preparation and review of a number of non-published nonlinear time history analysis studies. The ASCE/SEI 43-05 factors are as follows:

$$
\begin{aligned}
& \text { Sliding: } \boldsymbol{F} \boldsymbol{S}_{S}=3.0 \\
& \text { Rocking: } \boldsymbol{F} \boldsymbol{S}_{\boldsymbol{R}}=2.0
\end{aligned}
$$

\section{ASCE 4, SECTION 7.6 METHODOLOGIES}

As in ASCE/SEI 43-05, the new draft of ASCE 4 provides three general approaches to analyze rocking and sliding of unanchored components:

- Static Evaluation

- Conservatively Biased Reserve Energy

- Time History Analysis

Static Evaluation. The static evaluation is generally consistent with longstanding procedures established in building codes and in the NRC Standard Review Plan. Static evaluation entails an analysis (which generally considers dynamic effects and soilstructure interaction) to develop base shear and overturning moments, which are then multiplied by a safety factor of 1.1. These analyses usually assume the foundation is essentially rigid. Pressures applied to the foundation medium should be verified to be acceptable. If the resulting demand is less than the static resisting capacities, the 
component (or structure) will not slide or rock and no further evaluation is necessary. If the factored demands are greater than the static resistance capacities, then nonlinear analysis is required. Two general nonlinear approaches are described.

Reserve Energy Method. The simplified nonlinear approach provided in ASCE 4 is a reserve energy method, with formulations for both rocking and sliding. This method is well described in the appendices of ASCE/SEI 43-05 and elsewhere (Blume 1960, Yim et al. 1980, Aslam et al., 1980), and benchmarked against alternative approaches (Esfendari et al. 2009). It has been shown to be conservative with respect to more rigorous timeseries analysis (ASCE 2005). In ASCE/SEI 43-05, the generous conservatism of particularly the sliding analysis is credited by reducing the required general factor of safety from 3 , if a true best-estimate is developed, to 2 , if the conservatively biased reserve-energy method is used. In ASCE 4 the treatment of safety factors has been changed in general, as will be discussed later, and in particular for the reserve energy sliding analysis, there is no longer any distinction made between this conservative approach and a true best estimate. The reserve energy solutions for both sliding and rocking analyses are treated as best-estimates or mean solutions. These solutions assume rigid rocking or sliding bodies interacting with an undeformable foundation through a coefficient of restitution, and are only appropriate when such conditions approximately apply.

Time History Analysis. Two approaches to nonlinear time history analysis are provided; for each approach a number of sets of time histories matching the design basis earthquake (DBE) spectra and conforming to the requirements of Chapter 2 are required. In the first approach, 5 different time histories (sets) are applied to the same deterministic model. The mean of the 5 solutions is taken as the best estimate. In the second approach, a fully probabilistic solution modeling all significant sources of variability and uncertainty in the particular problem, including time histories, friction coefficients, dynamic stiffness of the support, stiffness of the object, damping, and geometric tolerances is generated. At least 16 independent realizations are required. The $80^{\text {th }}$ percentile non-exceedance probability (NEP) response is taken as the analytical demand. The time history problem may be solved in two or three dimensions, depending upon the relevant geometries.

Adjustment for Analytical Demand Conservatism. The intent of the standard is to produce analytical demands at about the $80 \%$ NEP level. The probabilistic time history analysis approach can directly generate this $80 \%$ NEP result. The deterministic approaches, reserve energy and the time history mean-of-5, generate results that are taken as mean or best estimates, and which require adjustment up to the $80 \%$ NEP level. This is achieved by multiplying the deterministic best estimate by a factor of 1.5 . This factor will be shown later in this paper to produce a reasonable estimate of the $80 \%$ NEP analytical demand.

Consideration of Uncertainties. Sliding and rocking problems are known to be sensitive to parameters which are usually not well-characterized. These may involve such phenomena as friction, surface irregularity, and phasing of input motions. To control and account for these effects, provisions are made for the consideration of uncertainties. For 
deterministic analysis, friction is to be taken at the 95\% NEP level for rocking, and at the $5 \%$ NEP level for sliding. Alternatively, pure rocking or pure sliding may be assumed. However, displacements due to rocking and displacements due to sliding need not be combined. For probabilistic analysis, friction is to be varied over an appropriate interval. Any combination of rocking and sliding may result and will factor into the reported displacement parameter.

Adjustment for Capacity Conservatism. To ensure the performance goals of ASCE/SEI 43-05 are satisfied, capacity factors precluding potential adverse interactions due to rocking or sliding are provided. Ideally these factors would be provided in ASCE/SEI 43-05 rather than in ASCE 4, because they have to do with capacity margin rather than demand margin. However, as an interim step that will allow future migration of the capacity factors to ASCE/SEI 43-05, ASCE 4 separates the demand factors from the capacity factors. A minimum capacity factor of 2.0 is required for both rocking angle and sliding displacement. The basis for this factor is judgment formed by familiarity with unpublished time history analyses results. Further development of a basis for these factors, particularly computed for the highly nonlinear rocking and sliding responses, may be an appropriate activity in support of the next revision of ASCE/SEI 43-05. However, based upon typical variability in seismic capacities as discussed in ASCE/SEI 43-05 Commentary, a factor of two will result in a capacity with approximately $98 \%$ probability of exceedance. In combination with the $80 \%$ NEP demand, this is expected to satisfy the ASCE 43-05 performance goal.

The combined demand and capacity factors result in a factor of safety of 3.0 relative to the best estimate. This is consistent with the factor of safety required for sliding in ASCE/SEI 43-05, and slightly higher than that previously required for rocking.

Response Limits. Solutions to rocking problems tend to become unpredictable at rocking angles approaching the stability limit. For example, it has been shown (DeJong 2009) that for ground motions which challenge gross stability, responses to multiple time histories with different phasing matching the same response spectrum can vary from less than $10 \%$ of the overturning angle all the way to overturning. Furthermore, this response is also shown to be sensitive to the duration of the shaking, which is not specified by a response spectrum.

To ensure a predictable response, the factored ( $80 \%$ NEP) rotation angle demand is limited to no more than $75 \%$ of the stability limit. For time history analysis, configurations which lead to any realization (either deterministic or probabilistic) overturning are prohibited. This prohibition is necessary for deterministic analysis based upon the mean response to 5 time histories because it would not be meaningful to average an overturned response with stable responses. A probabilistic analysis where some of the solutions result in overturning (when overturning is unacceptable) would be considered an "alternative methodology" as permitted in ASCE 4 Section 1.1.3.3. However, where, in general, alternatives to standard methodologies would need to demonstrate at least an $80 \%$ NEP, in the case of rocking and sliding ASCE 4 also provides the capacity factors required to achieve the ASCE/SEI 43-05 performance goals. 
Therefore, alternative rocking and sliding analyses in which some realizations overturn would need to demonstrate both a $1 \%$ or less probability of exceedance given the DBE, and a $10 \%$ or less probability of exceedance given 1.5 times the DBE. This would require a large number of solutions, depending in part upon the number of realizations which overturn.

An additional consideration applies to rocking of cylindrical components or of components whose bases have rounded cross sections. The reserve energy method accounts for dissipation of energy as the base impacts the foundation at each transition through the $0^{\circ}$ rotation angle. This coefficient of restitution-based energy dissipation is converted into an equivalent viscous damping, which is used to select the appropriately damped input response spectrum. However, it is possible that objects whose bases have circular cross sections may roll around the edge of their base when rocking. This type of response will exhibit much less energy dissipation than a pure rocking response, and the assumptions used to derive equivalent viscous damping are not accurate. To ensure a conservative analytical approach for components with circular bases, the rotation angle demand ( $80 \%$ NEP level) calculated by the simplified method (reserve energy) is limited to less than $10 \%$ of the angle at the stability limit. Detailed three dimensional nonlinear analysis can accurately model the rolling behavior of a round-based object, because energy dissipation is not dependent upon a linearization of the impact phenomenon (Sandia 2005). Therefore, when three dimensional time history analysis adequately models the potential rolling behavior of a component, the rocking angle limit remains $75 \%$ of the stability limit.

Summary of Methodologies. The nonlinear analysis methodologies and provisions are summarized in flowchart form in Figure 1. 


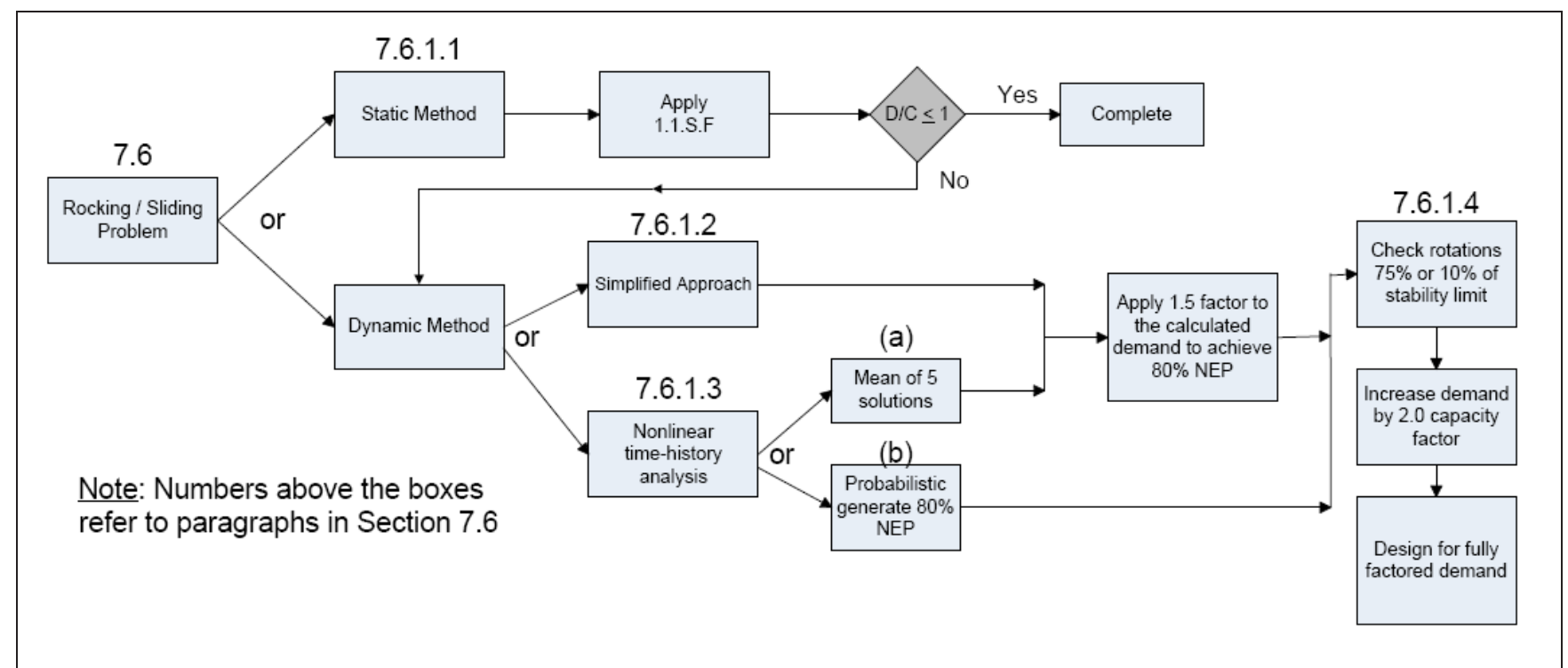

Figure 1: Summary of Rocking and Sliding Analysis Provisions 


\section{ADEQUACY OF ANALYTICAL DEMAND CONSERVATISM}

Standard Intent. The intent of the standard is to produce analytical demands at approximately the $80 \%$ NEP level, given the design basis earthquake (DBE). A key element in this approach is that the mean response, multiplied by the demand factor of 1.5 , will produce an approximately $80 \%$ NEP response.

Studies (Hutchinson and Chaudhuri 2006, Sandia 2005) have shown that rocking and sliding responses to various time histories matching a given demand response spectrum approximately follow a lognormal probability distribution. To the extent this formulation applies, a demand factor can be calculated from the assumed lognormal standard deviation, $\beta_{\mathrm{D}},\left(=\left(\beta_{\mathrm{R}}{ }^{2}+\beta_{\mathrm{U}}{ }^{2}\right)^{0.5}\right.$ where $\beta_{\mathrm{R}}$ and $\beta_{\mathrm{U}}$ are the logarithmic standard deviation for the inherent randomness and uncertainty, respectively, of the seismic response. The $80 \%$ NEP demand is given by the following equation,

$$
\frac{F_{D}}{F_{C}}=e^{0.84 \beta_{D}}
$$

where 0.84 is the standardized normal variable for the $20 \%$ (one-sided) exceedance probability level (that is, the $80 \% \mathrm{NEP}$ level), $\mathrm{F}_{\mathrm{D}}$ is the factored demand and $\mathrm{F}_{\mathrm{C}}$ is the calculated median demand, and $\beta_{\mathrm{D}}$ is the composite lognormal standard deviation of demand. Note that the factor thus calculated applies to the median value, which will be lower for a lognormal distribution than the mean value. Because ASCE 4 applies the demand factor to the calculated mean (or best estimate), Equation 1 is conservative. Values of $F_{D}$ corresponding to a range of values of $\beta_{D}$ are tabulated in Table 1 below.

Table 1. Example Values of $\mathbf{F}_{\mathrm{D}} / \mathbf{F}_{\mathbf{C}}$

\begin{tabular}{|c|c|}
\hline $\boldsymbol{\beta}_{\mathbf{D}}$ & $\mathbf{F}_{\mathbf{D}} / \mathbf{F}_{\mathbf{C}}$ \\
\hline 0.3 & 1.29 \\
\hline 0.35 & 1.34 \\
\hline 0.4 & 1.40 \\
\hline 0.45 & 1.46 \\
\hline 0.5 & 1.52 \\
\hline 0.55 & 1.59 \\
\hline 0.6 & 1.66 \\
\hline
\end{tabular}

As can be seen from Table 1, a demand factor of 1.5 will produce a reasonable estimate of the $80^{\text {th }}$ percentile demand for problems having lognormal standard deviations of about 0.5 or less, since the required multiplier in Table 1 is less than 1.5. 
Seismic response of components typically will have lognormal standard deviations in the range of about 0.2 to 0.4 (ASCE 2005); therefore, the factor of 1.5 provides a reasonable estimate of the demand at the $80 \%$ NEP level.

Rocking and Sliding Problems. However, rocking and sliding problems exhibit highly nonlinear and unique behavior. In this section new statistical processing of previously published results of a detailed three-dimensional study of the rocking response of cylindrical casks and rectangular storage modules using nonlinear time history analysis (NUREG/CR-6865, Sandia 2005) is reported. This will serve as an example to illustrate the variability of the responses in this class of problems and to test the adequacy of the ASCE 4 demand factor. NUREG/CR-6865 presents rocking results for casks and modules analyzed to three different standard spectral shapes, with five unique and spectrum-appropriate (magnitude, distance, tectonic environment) time history record sets fitted to each of the three spectra. The spectra are normalized to peak ground accelerations of $1.0 \mathrm{~g}$, and the time histories are scaled to various enveloping acceleration levels. For the cylindrical casks there are five acceleration levels, and for the rectangular casks there are four. Other parameters varied include foundation stiffness and coefficient of friction. Various output parameters are reported with analysis; only peak rotations are considered here.

Because of the relatively coarse binning of the model parameters, results from different friction sets and foundation stiffness sets are assumed here to represent distinct statistical populations. Therefore, the statistical analysis is performed on a series of results sets with only five results in each set. While this probably is an adequate sample size to define a mean, it is not considered adequate to define an $80 \%$ confidence interval. However, the large number of sample sets, each representing different permutations of friction and foundation stiffness, taken together are considered to provide a meaningful test of the adequacy of the ASCE 4 demand factor as applied to the rocking problem. Significantly, the results reported in NUREG/CR6865 show demonstrable rolling on the base by the cylindrical casks. In total, there are nine results sets (permutations of three friction coefficients and three foundation stiffnesses) for each spectral shape, each scaled acceleration level, and each component type. In total 243 sets of five results each were processed and are reported here.

Figure 2(a)-(f) below presents the ratios of the estimated $80^{\text {th }}$ percentile peak response to the mean peak response, calculated assuming lognormal distribution of the five results in each result set. There are two reasons why many of the sets are not shown in the figures. First, for low acceleration levels and particularly for the rectangular components, there was numerically zero variability (sometimes due to zero response), which precludes calculation of an $80^{\text {th }}$ percentile response level. Secondly, in cases of higher responses, some of the cylindrical components overturned, which precludes even calculation of the mean.

As can be seen in Figure 2, it is reasonably conservative to estimate the $80^{\text {th }}$ percentile rocking response by multiplying the mean of five time history responses by 
a demand factor of 1.5 in accordance with ASCE 4. These figures the result of additional statistical processing of the results published in NUREG/CR-6865).
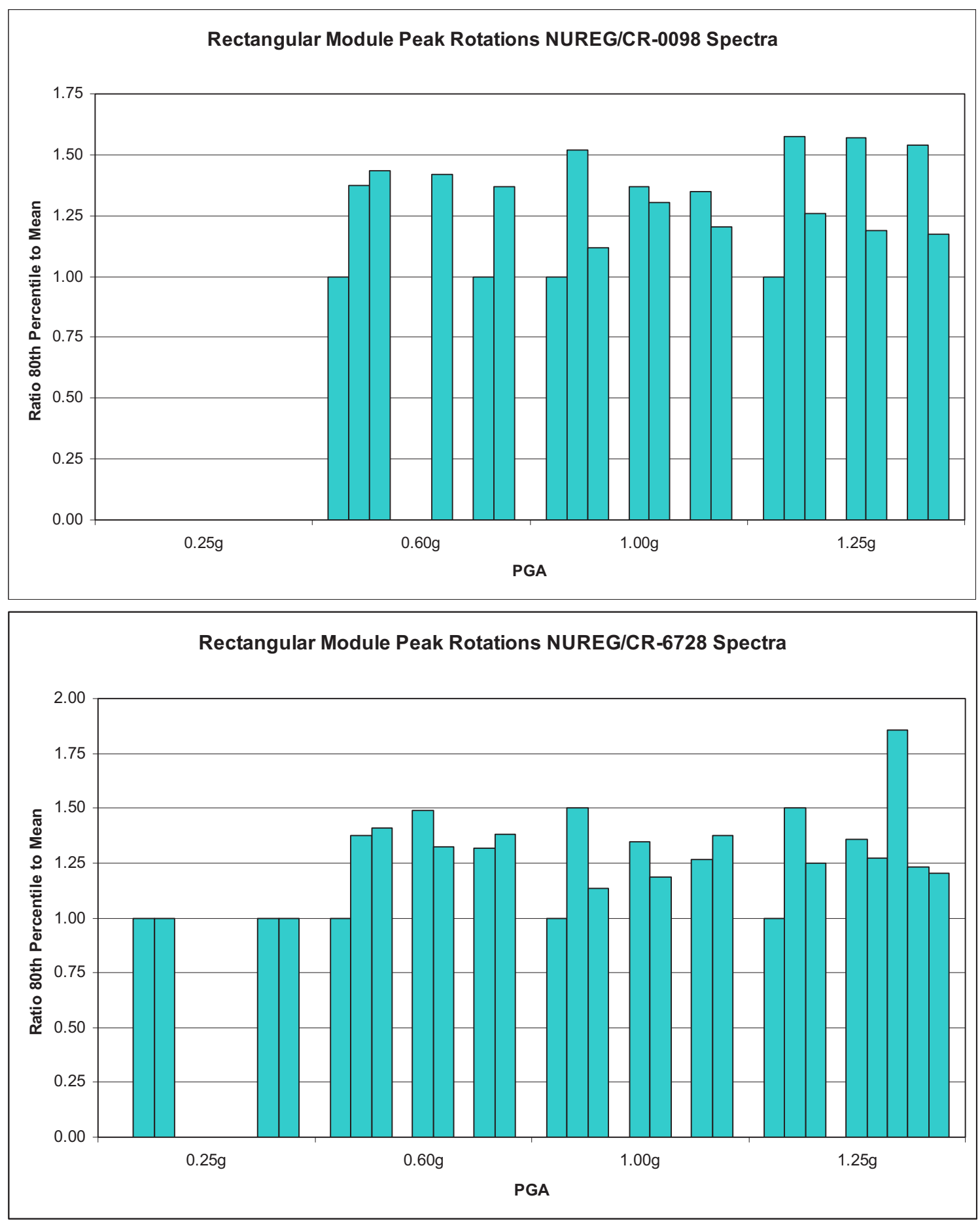

Figure 2(a) and (b): Rocking Response Ratio, $80 \%$ NEP to Mean 

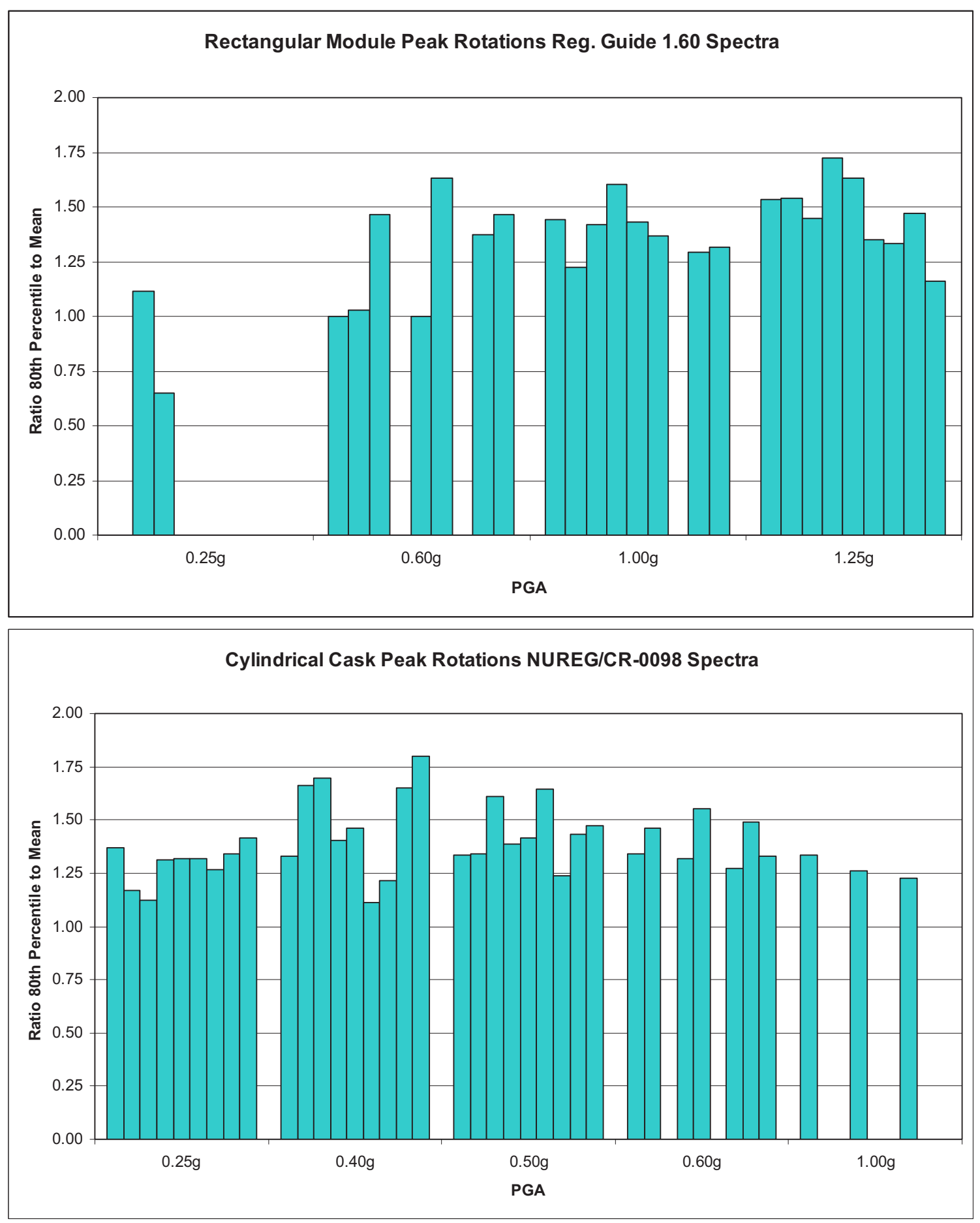

Figure 2(c) and (d): Rocking Response Ratio, $80 \%$ NEP to Mean 

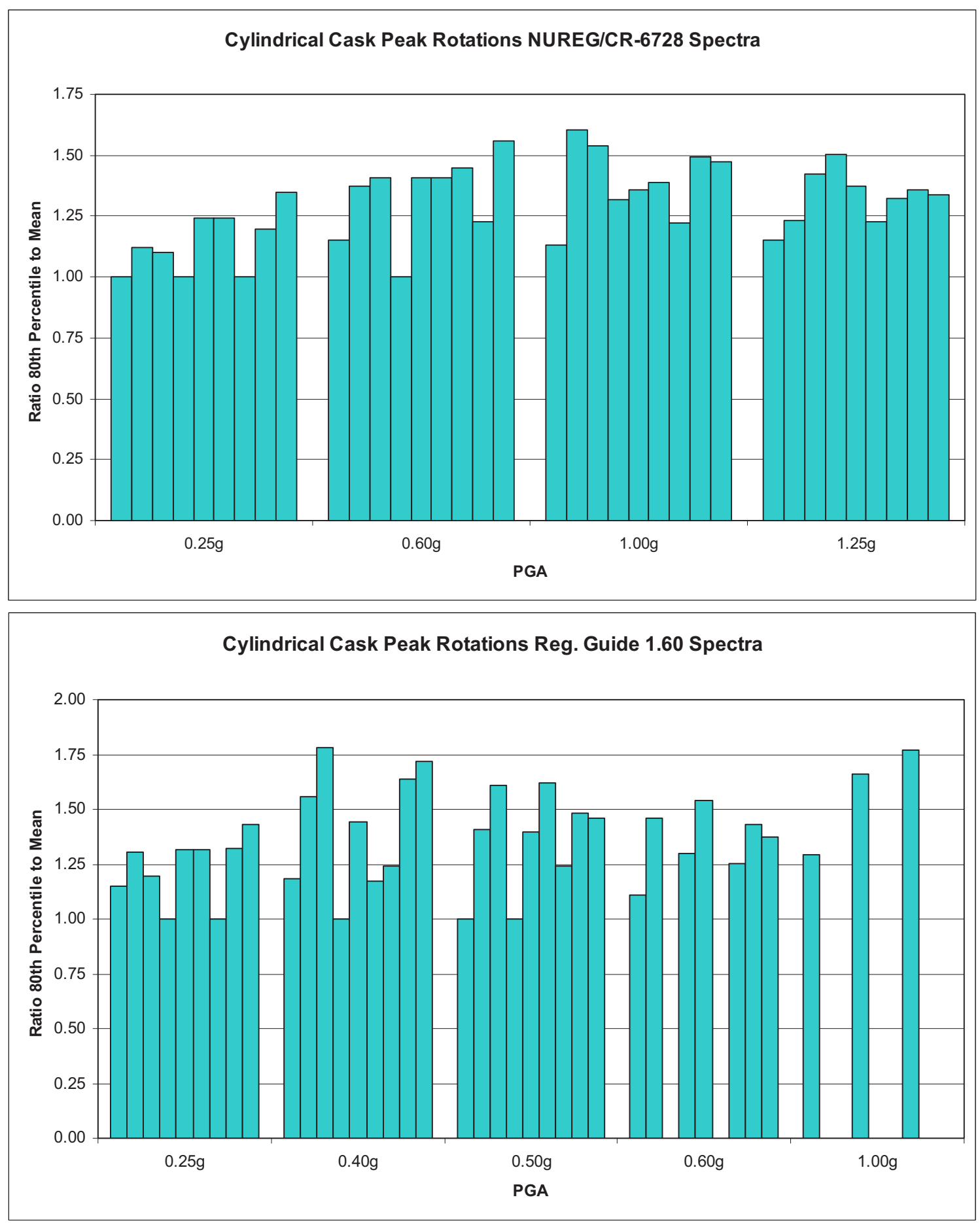

Figure 2(e) and (f): Rocking Response Ratio, $80 \%$ NEP to Mean

\section{CONCLUSION}

The new addition to ASCE-4 as Section 7.6 provides evolutionary guidance for seismic evaluation of rocking and sliding of unanchored bodies. Following the 
provisions of Section 7.6 will produce reasonably conservative estimates of the $80^{\text {th }}$ percentile rocking or sliding demands.

\section{ACKNOWLEDGEMENTS}

This manuscript has been authored by Battelle Energy Alliance, LLC under Contract No. DE-AC07-05ID14517 with the U.S. Department of Energy. The United States Government retains and the publisher, by accepting the article for publication, acknowledges that the United States Government retains a nonexclusive, paid-up, irrevocable, world-wide license to publish or reproduce the published form of this manuscript, or allow others to do so, for United States Government purposes.

\section{REFERENCES}

American Society of Civil Engineers, ASCE 4-98, Seismic Analysis of Safety-Related Nuclear Structures: Standard and Commentary, 1998.

American Society of Civil Engineers, ASCE 4-Rev 2, Seismic Analysis of SafetyRelated Nuclear Structures: Standard and Commentary, Pending 2011.

American Society of Civil Engineers, ASCE/SEI 43-05, Seismic Design Criteria for Structures, Systems and Components in Nuclear Facilities, 2005.

Aslam, M, Godden, W.G., and Scallse, D.T. "Earthquake Rocking Response of Rigid Bodies," Journal of Structural Engineering, 1980.

Blume, J.A., "A reserve energy technique for the earthquake design and rating of structures in the inelastic range." Proceedings of the Second World Conference on Earthquake Engineering, Tokyo and Kyoto, Japan, 1061$1083,1960$.

Dejong, M. J., "Redefining the Vulnerability of Rocking Structures to Horizontal Ground Motion" c.2009.

Esfandiari S., Wandell, Christopher, "Comparison of Approximate Methods for Sliding and Rocking Evaluation of Unanchored Platforms", SMiRT 2009.

Hutchinson, T .R. and S. R. Chaudhuri, "Simplified Expression for Seismic Fragility Estimation of Sliding-Dominated Equipment and Contents, " Earthquake Spectra, Volume 22 No. 3, pages 700-732, August 2006.

Sandia National Laboratories, NUREG/CR-6865, Parametric Evaluation of Seismic Behavior of Freestanding Spent Fuel Dry Cask Storage Systems, 2005.

Yim, S.C.-S., Chopra, A.K., and Penzien, J. "Rocking response of rigid blocks to earthquakes." Earthquake Engineering and Structural Dynamics, Vol. 8, 565$580,1980$. 